\title{
Some Harmonic Properties of an Oscillating Fabry-Perot Interferometer
}

\author{
M. Gadsden* and H. M. Williams*
}

(March 9, 1966)

\begin{abstract}
The transmission of a spectral line through an air spaced Fabry-Perot interferometer is considered. The harmonic components of the transmission when the spacing of the interferometer is oscillated are discussed and it is found that the components can be used in a servocontrol system to offset drifts in the optical thickness of the interferometer. A possible use of such a controlled system in observations of the night airglow is discussed.
\end{abstract}

Key Words: Fabry-Perot interferometer, airglow photometry.

\section{Introduction}

In two recent papers, Mielenz et al. [1, 2], have discussed Fabry-Perot interferometers which use plates whose separation can be controlled electrically. The spacers in these interferometers are made from a piezoelectric ceramic material, lead zirconate-titanate, and the plate separation is thus directly, and easily, controllable by the application of an electric potential across two faces of the material. Mielenz et al., (loc. cit.) present full details of the use of this material and discuss the advantages and some applications of this piezoelectric scanning technique; it is the purpose of this paper to point out that the harmonic output of such a system has some advantages in the servocontrolling of the spacing of an interferometer.

The use of oscillating piezoelectric spacers to control the spacing and parallelism of a Fabry-Perot interferometer was first described by Ramsay [3]. In Ramsay's system, two passes of the interferometer by white light are used, and equality and constancy of the path length in the interferometer along the two light beams is maintained by seeking the maximum transmission for white light. The servocontrol to seek this maximum involves a minute superposed oscillation to give an error signal. The servocontrol to be described in what follows is similar to Ramsay's system except that the control for constancy of interferometer spacing is achieved from the spectral emission being observed with the interferometer, and does not demand subsidiary white light illumination. ${ }^{*}$ Institute of Telecommunication Science and Aeronomy, Environmental Science Serv.
ices Administration, Boulder Colo. Formerly the Central Radio Propagation Laboratory of the National Bureau of Standards.

\section{Harmonic Analysis of the Output}

As is well known [4], the transmission at normal incidence of a perfect Fabry-Perot interferometer is given by the Airy relation

$$
\frac{I}{I_{m}}=\frac{1}{1+F \sin ^{2}(2 \pi D)}
$$

where $D=$ optical thickness, in wavelengths, of the interferometer i.e., half the order,

$I=$ transmitted intensity of a spectral line, whose width is much less than the instrumental width;

$I_{m}=$ transmitted intensity when $D=\frac{n}{2}, n$ being and $F=$ a constant, dependent on the reflectivity of the coatings on the interferometer plates.

$F$ is related to the finesse, $\mathscr{F}$, of the interferometer by the relation [4]

$$
F=\left(\frac{2 \mathscr{F}}{\pi}\right)^{2}
$$

If a sinusoidal potential be applied to the piezoelectric spacers of an interferometer, $D$ in expression (1) will become $\left(d_{0}+d \cos 2 \pi f t\right)$, where $f$ is the frequency of the alternating potential and $d$ is half the total excursion in length of the spacers. In these circumstances, $I / I_{m}$ becomes a periodic function of time, $t$, with a period equal to $1 / f$. The function, $I / I_{m}$, will contain a series of harmonics of $f$; we shall direct our attention to the amplitudes of the zeroth, first and second harmonics in what follows, with the convention that the $n$th harmonic has the frequency $n f$. 
The harmonic amplitudes and phases contained in $I / I_{m}$ will vary as $2 d_{0}$ is an integer or not. This is readily seen from figure 1 , in which is sketched, from top to bottom,

(i) The variation of $I / I_{m}$ as a function of plate separation, $d_{0}$;

(ii) two spacer oscillations, (A) and (B), with $d=1 / 2$ and $d_{0}$ at the positions indicated;

(iii) the outputs resulting from the applied spacer oscillations, and

(iv) two more outputs resulting from spacer oscillations with $d=1 / 2$ and $d_{0}$ at the marked positions, $(\mathrm{C})$ and (D).

It is readily seen that the second harmonic predominates in (A) and (B) and that the first harmonic predominates in (C) and (D). Photographs of oscilloscope traces showing approximations to these conditions are shown in figure 2 ; note, in both figure 1 and figure 2 , that there is a change in phase of the output between (C) and (D).
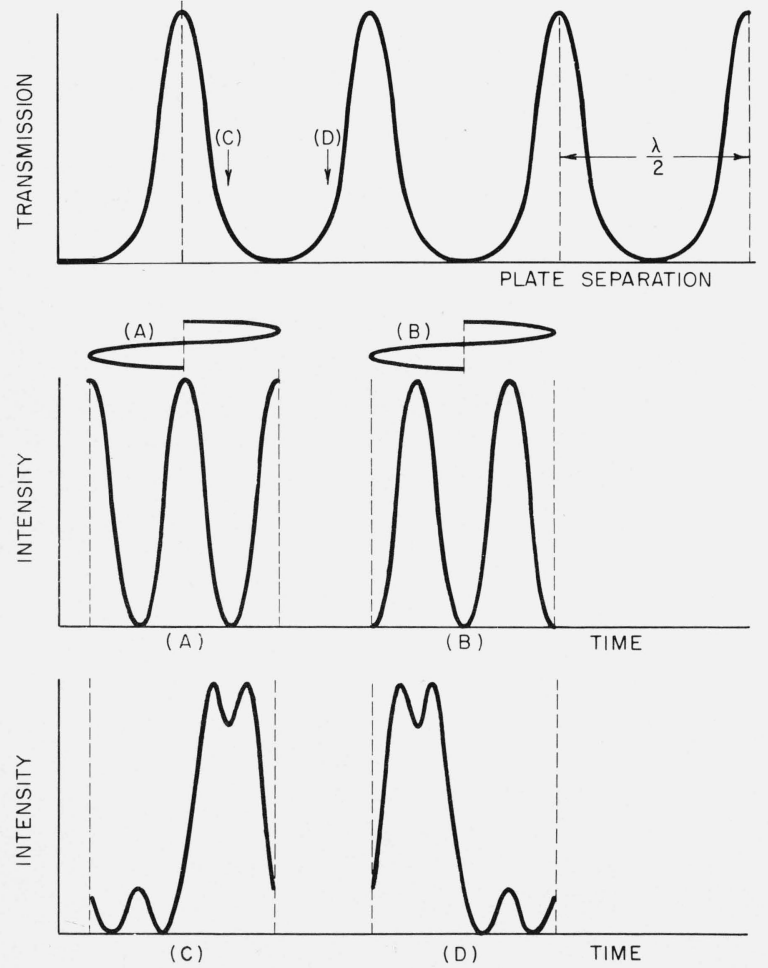

FIGURE 1. Top: the variation in transmission of monochromatic radiation by a Fabry-Perot interferometer, as the separation of the plates is increased.

Middle: the time variation of the transmitted intensity when the plate separation is oscillated over the ranges indicated by (A) and $(\mathrm{B})$.

Bottom: the time variations resulting from spacer oscillations equal in amplitude to $(A)$ and $(B)$, but centered at the points $(C)$ and $(D)$ indicated in the top sketch.
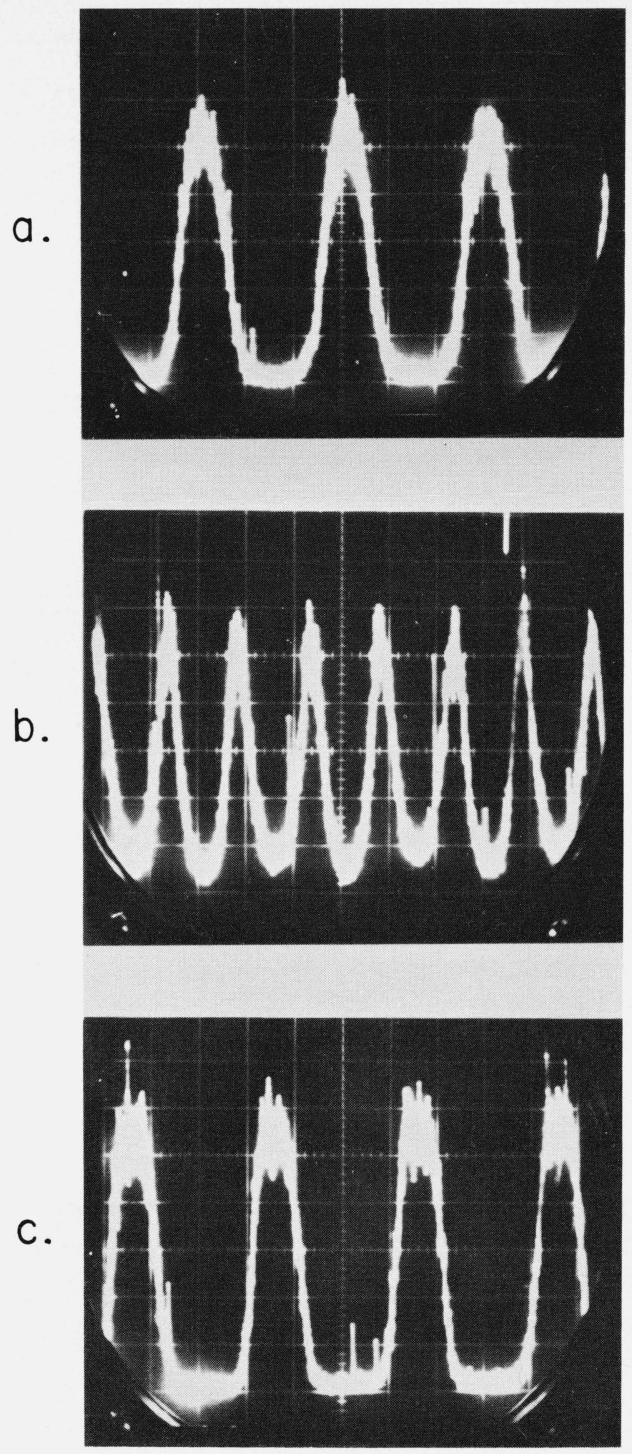

FIGURE 2. Oscillograms of an oscillating interferometer corresponding to three settings of the mean spacing.

Numerical harmonic analysis of $I / I_{m}$ has given the results shown in figure 3 . The amplitudes of the harmonics depend on whether $2 d_{0}$ is an integer, i.e., if we write

$$
d_{0}=\frac{1}{2}(n+\epsilon),
$$

where $\epsilon<1$ and $n$ is integral, all possible variations of $d_{0}$ are covered by varying $\epsilon$. In figure 3 , therefore, the abscissae are given in terms of $\epsilon / 2$, with the limits $0,+0.5$.

The upper plot in figure 3 is for an interferometer with a finesse of 10 , and $d$ equal to 0.25 . With this amount of driving potential, the point of interest is that the second harmonic is a maximum at the value of $\epsilon$ 

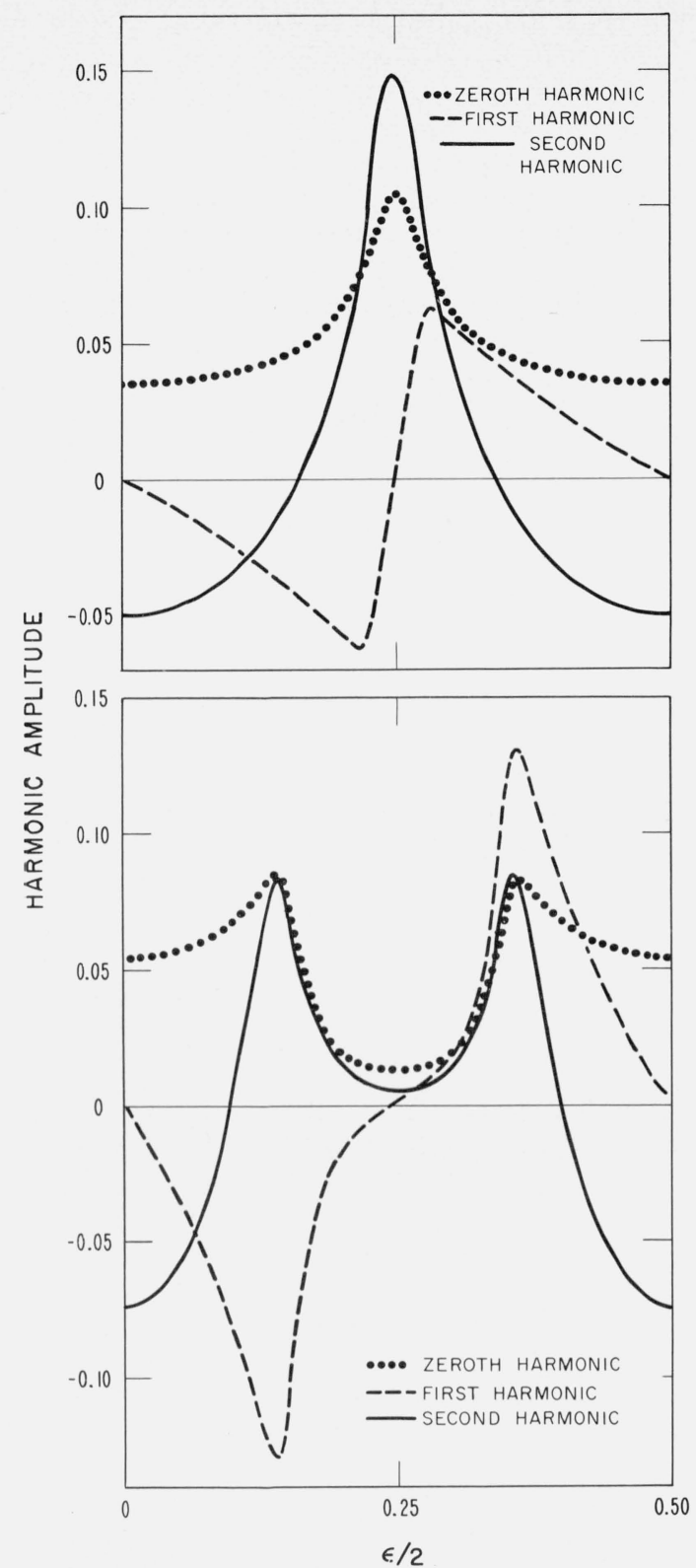

FIGURE 3. Harmonic amplitudes of transmitted monochromatic radiation as a function of the departure of the mean spacing from an integral of half-wave lengths.

where the first harmonic is zero (and where, also, it changes phase). This, because of the symmetry of $I / I_{m}$, holds for all values of the finesse so long as $d=0.25$. When $d$ is either increased or decreased, the null point of the first harmonic remains at $\epsilon / 2=0.25$, but the second harmonic shows two maxima at progressively increasing distances from $\epsilon / 2=0.25$. (When $d$ is increased to 0.5 , then the two maxima of the second harmonic recombine at $\epsilon / 2=0,0.5$, where the first harmonic has another null point.) The lower plot in figure 3 shows the effect of reducing $d$ to 0.15 .
This property of the second harmonic going to a maximum where the first harmonic is zero and changing phase, is of immediate application in using a servosystem to control the mean optical thickness, $d_{0}$, of the interferometer. $d_{0}$ is related to the metric thickness, $d^{*}$, of the air layer between the interferometer plates by

$$
d_{0}=\frac{\mu d^{*}}{\lambda_{0}}
$$

in which $\mu$ is the refractive index of the air in the interferometer and $\lambda_{0}$ is the wavelength in vacuo of the observed emission line. $\mu$ is, of course, dependent on the temperature and pressure of the air and, in the course of time, can be expected to show small changes; more important, however, is the temperature dependence of $d^{*}$ through the thermal expansion of the spacer material. It is easy, however, to compensate for these changes in $d^{*}$ and $\mu$ by arranging for a bias voltage to be applied to the spacer material from a servosystem which seeks the null of the first harmonic. With a tuned system responding to the second harmonic, one then has a controlled interferometric photometer for monitoring the radiance in a spectral line.

In this connection, it is intuitively obvious that there will be a particular value for the finesse that gives the most output in the second harmonic. If the finesse is very high, the proportion of the cycle during which the spectral line is passed by the interferometer will be small, relatively more of the available signal appearing in the higher harmonics. As the finesse is decreased, the output signal will tend to become more and more sinusoidal, although with a finesse equal to zero-i.e., with no interferometer-there will be no second harmonic. Somewhere, therefore, there will be a maximum in the curve of the second harmonic amplitude versus finesse; figure 4 shows this maximum to be close to a finesse of six.

In the above analysis, it has been assumed that the interferometer is 'perfect,' with $I / I_{m}$ given by (1). In practice, the performance of an interferometer falls short of perfection due to surface imperfections in the plates, lack of perfect adjustment in parallelism, and the use of a finite scanning aperture in the plane of the fringes. Measurements we have made using a prototype system, with a finesse equal to approximately five, have shown that the amplitude and phase relationships between the harmonics, derived above, hold for a practical system.

It has also been found, during these measurements, that the ceramic material shows a 'damped' response to the application of a potential, such that if a potential be switched on, the majority of the strain appears very rapidly but the final strain appears only after the lapse of a few seconds. This implies that the voltage excursion required to produce a given excursion in length of the spacers will be a function of the frequency of an applied alternating potential, and will 


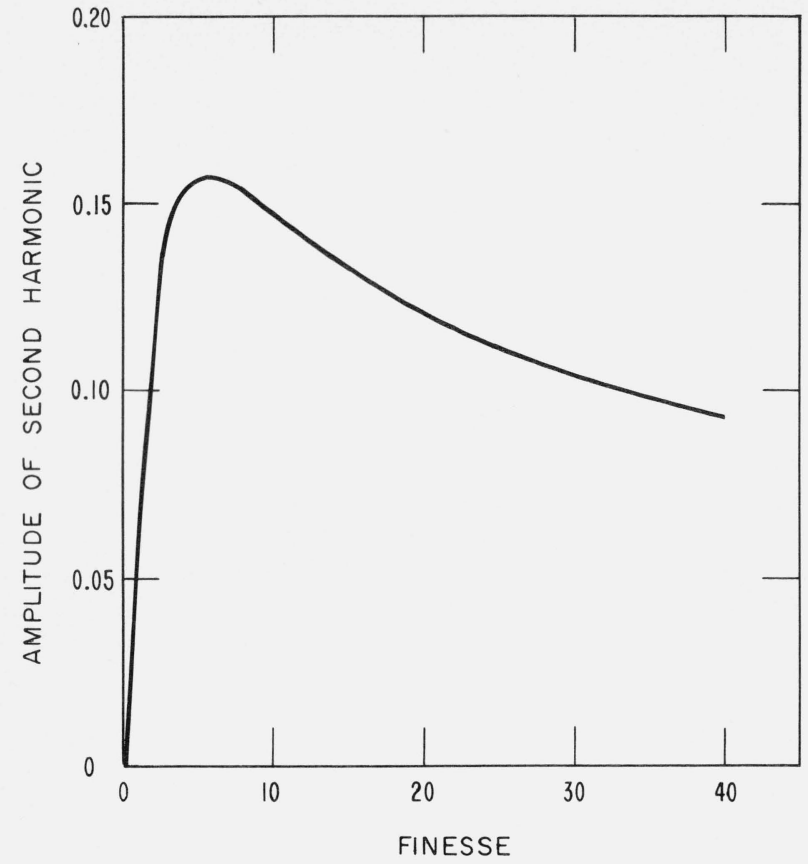

FIGURE 4. Change of the maximum of the second harmonic with change in the finesse of the interferometer.

be somewhat greater than that indicated from quasistatic measurements. Since the first harmonic null point is independent of the amplitude of the alternating driving potential, a practical procedure of setting the bias and driving potentials is to set first the bias potential to give zero first harmonic and then to vary the amplitude of the superposed alternating potential to maximize the second harmonic. We have found, using our prototype system, that this procedure is quick, and reliable.

\section{Airglow Photometry}

The problem basic to photometric determination of the night airglow is that of observing an atomic emission line spectrally embedded in a continuum and emission bands. Two basic techniques are in use to eliminate, or take account of, the radiation spectrally surrounding an emission line. The most frequently used is that of 'two-filter' photometry, in which interference filters are used either to measure the radiance of the continuum in a spectral region free of atomic lines so that the contamination of the atomic line measurement may be estimated (Roach and Barbier [5]) or, in a method due to Barbier [6], to make two measurements centered on the emission line with differing proportions of background contamination. The first of these methods suffers from the uncertainty in knowledge of the spectral distribution of the background radiation (Truttse [7, 8]); both 'two-filter' methods use pairs of observations sequential in time, and are thus inherently unsuited for use in observations involving dynamic scanning of the sky. In this case, one uses the second basic technique of extracting an atomic emission line from the continuum, which is to use either a birefringent filter (Koomen et al. [9]), or a rocking interference filter (Filosofo et al., [10]). The calibration of a birefringent-filter photometer presents some difficulties although these are not insuperable (Smith and Alexander [11]; Dandekar et al., [12]).

Let us consider the basic problem. When a photometer is pointed at the night sky, the response, $R$, consists of two parts

$$
R=R_{b}+R_{e},
$$

where $R_{b}$, the response to the background continuum, is given by

$$
R_{b}=W \cdot I_{b} \cdot S
$$

( $W=$ equivalent width of the filter, $I_{b}=$ specific spectral radiance of the continuum and $S=$ spectral response of the photocell). Similarly, $R_{e}$, the response to the emission line, is given by

$$
R_{e}=T_{e} \cdot I_{e} \cdot S
$$

( $T_{e}=$ transmission of the filter at the wavelength of the emission line and $I_{e}=$ radiance of the line.)

If we now take an oscillating interferometer as the filter in the photometer, then $T_{e}$, given by (6) above, becomes of the form $I / I_{m}$ discussed above; particularly, the first harmonic in $R_{e}$ can be used to compensate for thermal changes in the spacer length and the second harmonic used to determine $I_{e}$. Under these conditions, the contamination of the measurement by $R_{b}$, eq (5), can become extremely small: first, by making $W$ very small, as is easily done with an air-spaced interferometer, and, second, because the second harmonic in $R_{b}$ will depend only on the derivatives of $\left(I_{b} \cdot S\right)$, taken at the emission wavelength, of even degree. These derivatives will be appreciable only if the emission line lies in a fraunhofer line in the continuum, or is close to emission band structure.

This result is seen by expanding $I_{b}$ in Taylor's series, ignoring the variations of $S$ with $\lambda$ :

$$
\begin{aligned}
I_{b}\left(\lambda_{0}+\Delta \lambda \cos \mathrm{wt}\right)=I_{b}\left(\lambda_{0}\right) & +\Delta \lambda \cos \mathrm{wt}\left(\frac{d I_{b}}{d \lambda}\right)_{\lambda=\lambda_{0}} \\
& +\Delta \lambda^{2} \cos ^{2} \mathrm{wt}\left(\frac{d^{2} I_{b}}{d \lambda^{2}}\right)_{\lambda=\lambda_{0}}+\ldots
\end{aligned}
$$

where $\left(\lambda_{0}+\Delta \lambda \cos w t\right)$ expresses the oscillating wavelength of the transmission peak of the interferometer pass-band. (It is to be noted that the coefficient of $\cos 2$ wt contains only $\frac{d^{2} I_{b}}{d \lambda^{2}}, \frac{d^{4} I_{b}}{d \lambda^{4}}, \ldots$ etc.) 
It should be noted that the presence of appreciable odd derivatives in $\left(I_{b} \cdot S\right)$ will give a contribution to the first harmonic signal, causing the servosystem to seek a null point away from the peak of the second harmonic. In the presence of a serious background continuum, therefore, it would be best to make $W$ as small as convenient.

\section{Conclusions}

A system such as the one outlined above offers the possibility of continual control of the spacing, while observations of a feeble spectral line are in progress. The use of three small spectral line sources, sampling the interferometer at three separated positions, is an obvious extension of this monitoring to the control of parallelism. In this case, we have a system closely resembling that of Ramsay [3], but one which does not demand an optical system, with close tolerances, to return the sampling light for a second pass through the interferometer.

\section{References}

[1] Mielenz, K. D., R. B. Stephens, and K. F. Nefflen, J. Res. NBS 68C (Engr. and Instr.) No. 1, 1, (1964).

[2] Mielenz, K. D., J. Res. NBS 68C (Engr. and Instr.) No. 2, 73, (1964).

[3] Ramsay, J. V., Appl. Optics 1, 411 (1962).

[4] Born, M. and E. Wolf, Principles of Optics (Pergamon, New York, 1964).

[5] Roach, F. E. and D. Barbier, Trans. Amer, Geophys. Union 31, 7, (1950).

[6] Barbier, D., The Airglow and the Aurorae, ed. E. B. Armstrong and A. Dalgarno (Pergamon, New York, 1955).

[7] Truttse, Yu. L., Section 4, No. 10, p. 5, IGY Program, Moscow (1963).

[8] Truttse, Yu. L., Section 4, No. 10, p. 8, IGY Program, Moscow (1963).

[9] Koomen, M. J., C. Lock, D. M. Packer, R. Scolnik, and R. Tousey, J. Opt. Soc. Amer. 44, 827, (1954).

[10] Filosofo, I., J. A. Greenspan, and C. M. Groom, Appl. Optics 4, 215 (1965).

[11] Smith, L. L. and R. B. Alexander, IQSY Instruction Manual No. 5, Airglow. p. 25; IQSY Secretariat, London, 1963.

[12] Dandekar, B. S., J. C. Pomalaza, and W. F. Bellew, Appl. Optics 4, 295 (1965).

(Paper 70C3-226) 